\title{
Subcutaneous Versus Intraperitoneal Administration of Insulin on Post-Prandial Hyperglycaemia and Glucose Turnover in Alloxan Diabetic Dogs
}

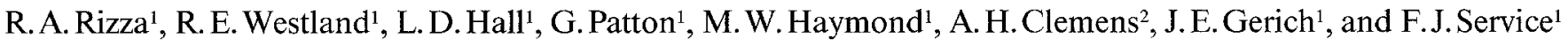 \\ ${ }^{1}$ Endocrine Research Unit, Departments of Medicine and Physiology, Mayo Medical School and Mayo Clinic, Rochester, Minnesota, and \\ ${ }^{2}$ Life Science Instruments, Miles Laboratories, Elkhart, Indiana, USA
}

Summary. The effects of subcutaneous and intraperitoneal insulin delivery by a closed-loop insulin infusion device on post-prandial hyperglycaemia and rates of glucose appearance and disappearance were compared in alloxan diabetic dogs. No differences in basal or post-prandial values or patterns of response were observed between the two routes of insulin delivery. In addition, the amounts of insulin infused and the plasma insulin concentrations achieved were not different for the two routes of insulin administration. These studies demonstrate that in the dog there appears to be no difference in the pattern of disposal of glucose from a mixed meal when insulin was administered intraperitoneally or subcutaneously at the rates of insulin infusion used in these experiments.

Key words: Subcutaneous insulin, intraperitoneal insulin, glucose turnover, diabetic dogs.
The development of open-loop (preprogrammable) insulin infusion devices has made achievement of euglycaemia in insulin-dependent diabetic patients a realistic goal. The best route for insulin administration by such devices has not yet been established. Although close to normal plasma glucose concentrations have been achieved in ambulatory diabetic subjects using the peripheral venous route for insulin administration, longterm use of this route has been limited because of the risks associated with maintenance of chronic venous access [1-5]. Because of ease of access and safety, the subcutaneous route has been widely used for insulin administration by open-loop insulin infusion devices [6-12]. Although excellent glycaemic control has been achieved with this route, it has the disadvantage of delayed and sometimes variable insulin absorption. Furthermore, both the peripheral venous and subcutaneous routes of insulin administration deliver insulin into the peripheral circulation rather than into the portal venous circulation, which is the physiological route. This has usually resulted in peripheral hyperinsulinaemia $[8,13]$. In addition, since the liver is a major organ of glucose disposal in the post-prandial state in man, it is possible that the pattern of glucose clearance (e.g. hepatic versus extrahepatic) may differ when insulin is infused into a peripheral rather than a portal vein. Since direct access to the portal vein is currently not feasible in man, several investigators have advocated intraperitoneal infusion because of its large absorptive surface and potential for direct absorption of insulin to the portal circulation [14-18]. However, because access to the peritoneal cavity is associated with more discomfort and greater morbidity than the subcutaneous route, a definite therapeutic advantage of the intraperitoneal route over the subcutaneous route needs to be demonstrated to justify its use.

Since similar plasma glucose profiles can be observed in the presence of marked differences in the associated rates of glucose production and ultilization, merely measuring plasma glucose concentrations may fail to detect important differences in the pattern of glucose disposal. The present studies were undertaken, therefore, to determine whether the pattern of glucose disposal following a mixed meal differed between intraperitoneal and subcutaneous insulin administration. For this purpose, the rates of glucose appearance, disappearance and clearance, as well as plasma glucose and free insulin concentrations, were measured following a standard mixed meal in alloxan diabetic dogs when insulin was given intraperitoneally and subcutaneously.

\section{Methods}

Five adult mongrel alloxan diabetic dogs $(15-20 \mathrm{~kg})$ who were demonstrated to be severely insulin deficient (marked hyperglycaemia and ketonuria on insulin withdrawal) underwent placement of a Thomas gastrostomy under general anaesthesia (pentobarbitol sodi- 

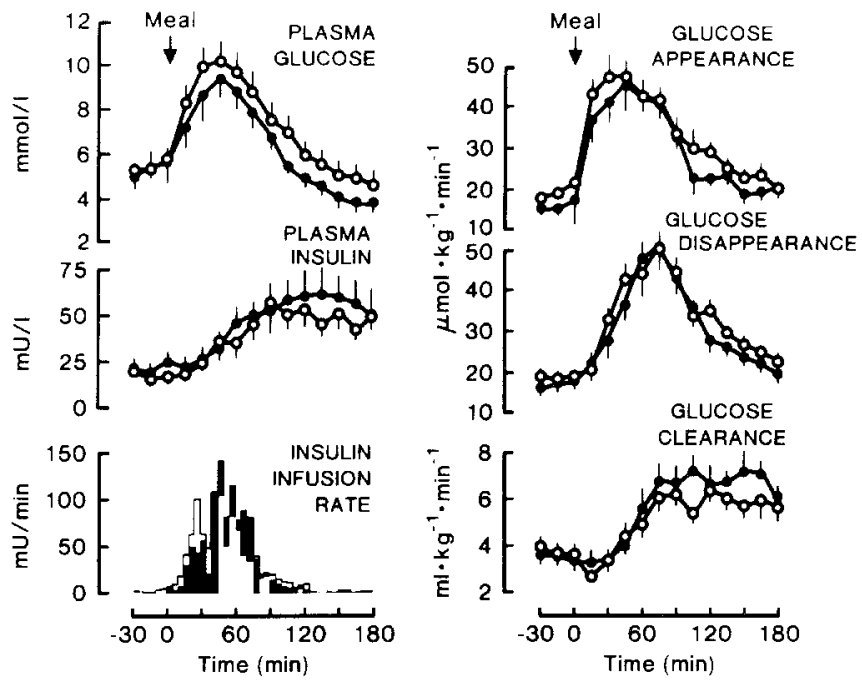

Fig. 1. Comparison of effects of the subcutaneous and intraperitoneal routes of insulin administration by the 'Biostator' on plasma glucose, plasma insulin and rates of glucose appearance, glucose disappearance and glucose clearance following a mixed meal in five diabetic dogs. Insulin infusion rates are given per 5 min intervals. $O$ and bars $=$ intraperitoneal insulin; and bars = subcutaneous insulin.

Values expressed as mean $\pm \mathrm{SEM}$

um, $28 \mathrm{mg} / \mathrm{kg}$, IV). The dogs were permitted a recovery period of not less than 2 weeks after the surgery before any studies were performed. Each animal was treated with sufficient intermediate-acting insulin (Lente 17-22 units, Eli Lilly, Indianapolis, Indiana, USA) as a single morning injection to maintain fasting plasma glucose concentrations between 7-14 mmol/1, except on the morning of an experiment when it was withheld. All animals were fed a standard canine diet (Nutrena, Minneapolis, Minnesota, USA). Each dog was studied twice after $12 \mathrm{~h}$ of fasting, during which insulin $(0.6 \mathrm{U} / \mathrm{ml})$ was infused by 'Biostator glucose controlled inuslin infusion system' to control prandial glycaemia on one occasion using the subcutaneous route and on the other the intraperitoneal route. Standard algorithm constants were used on both occasions. These algorithms were utilized since preliminary experiments demonstrated that they resulted in comparable insulin infusion profiles and comparable glycaemic control when infusing insulin intraperitoneally or subcutaneously. On the morning of each experiment, short No.18 gauge catheters (Jelco, Raritan, New Jersey, USA) were placed percutaneously into a vein in each foreleg, one for infusion of isotope and insulin, the other for blood withdrawal for continuous glucose monitoring (Biostator). A third catheter (Desert Pharmaceuticals, Sandy, Utah, USA) was placed in a hindleg vein and advanced to the inferior vena cava for intermittent blood sampling. When the intraperitoneal route was used, a No.18 gauge plastic catheter (Jelco, Raritan, New Jersey, USA) was inserted $4 \mathrm{~cm}$ into the peritoneal cavity percutaneously in the midline of the lower anterior abdominal wall; its presence in the peritoneal cavity was verified before and after each experiment by injecting saline and obtaining no return of either saline, blood, urine, or intestinal contents. When the subcutaneous route was used, a No.23 gauge 'butterfly' needle was inserted subcutaneously in the interscapular area. Euglycaemia was achieved and maintained by the Biostator for $2 \mathrm{~h}$ before the meal. During this period, a primed $(5.5 \mu \mathrm{Ci})$ continuous $(0.05 \mu \mathrm{Ci} /$ min) infusion of $\left[3-{ }^{3} \mathrm{H}\right]$ glucose (New England Nuclear, Boston, Massachusetts, USA; specific $17.54 \mathrm{Ci} / \mathrm{mmol} / 1$ made up in $0.154 \mathrm{mmol} / 1$ saline solution, $25 \mu \mathrm{Ci} / 50 \mathrm{ml}$ ) was begun. Two hours were permitted for isotope equilibration. After collection of appropriate blood samples for baseline determinations, a standard liquid meal [10 Kcal $/ \mathrm{kg}$, Compleat B] (Doyl Pharmaceuticals, Minneapolis, Minnesota, USA) was given per gastrostomy over a $2 \mathrm{~min}$ interval. Blood samples were obtained at $15 \mathrm{~min}$ intervals for $180 \mathrm{~min}$ following the meal.

\section{Laboratory Methods}

Samples for insulin determinations [19] were collected in chilled $4 \mathrm{ml}$ tubes containing $17 \mathrm{mg}$ EDTA (Sigma, St.Louis, Missouri, USA). Blood for plasma glucose and glucose specific activity was collected in NaF-oxalate tubes (Kimble-Terumo, Elkhart, Indiana, USA) and an aliquot of this plasma was used for determination of glucose concentration in duplicate (Yellow Springs Instruments 23 A). Plasma $\left[3-{ }^{3} \mathrm{H}\right]$ glucose specific activity was determined as described previously [20]. In brief, triplicate $0.3 \mathrm{ml}$ aliquots of deproteinized plasma were evaporated to dryness at $37^{\circ} \mathrm{C}$ under compressed air to remove tritiated water. The residue was resuspended in $0.5 \mathrm{ml}$ distilled water and after the addition of $10 \mathrm{ml}$ Aquasol (New England Nuclear, Boston, Massachusetts, USA), its radioactivity was counted in a refrigerated liquid scintillation spectrometer. Correction for quenching was made using the method of external standard ratios. The calculated infusion rate of isotope was verified by measuring the volume of the $\left[3-{ }^{3} \mathrm{H}\right]$ glucose before and after each experiment.

Rates of glucose appearance and disappearance were calculated using the equations of Steele et al. [21], as modified by De Bodo et al. [22]. The validity of these equations for nonsteady state conditions has been previously discussed in detail [23]. Glucose clearance was calculated by dividing the rate of glucose disappearance by the appropriate plasma glucose concentration [24]. The use of 3-H-3 glucose as a nonrecycling trace has been previously established [25].

All the data are expressed as mean $\pm \mathrm{SEM}$, and their statistical significance was evaluated using Student's two tailed paired (and when appropriate, unpaired) t-test [26].

\section{Results}

Before the mixed meal, baseline values for plasma glucose $(5.1 \pm 0.6$ and $5.3 \pm 0.4 \mathrm{mmol} / \mathrm{l})$, plasma insulin $(22 \pm 4$ and $16 \pm 1 \mathrm{mU} / 1)$ and rates of glucose appearance $\left(16.7 \pm 2.2\right.$ and $\left.20.6 \pm 3.9 \mu \mathrm{mol} \cdot \mathrm{kg}^{-1} \cdot \mathrm{min}^{-1}\right)$, disappearance $(16.7 \pm 1.1$ and $18.9 \pm 1.7$ $\left.\mu \mathrm{mol} \cdot \mathrm{kg}^{-1} \cdot \mathrm{min}^{-1}\right)$ and clearance $(3.3 \pm 0.3$ and $3.7 \pm$ $0.4 \mathrm{ml} \cdot \mathrm{kg}^{-1} \cdot \mathrm{min}^{-1}$ ) were not significantly different for the subcutaneous and intraperitoneal routes of insulin administration, respectively (Fig. 1). Following the mixed meal, changes in plasma glucose and plasma insulin concentrations were comparable for the subcutaneous and intraperitoneal routes of insulin administration. Neither peak concentrations $(9.3 \pm 1$ versus $10.1 \pm 1 \mathrm{mmol} / \mathrm{l}$, glucose; $66 \pm 16$ versus $57 \pm$ $10 \mathrm{mU} / 1$, insulin) nor total areas under the curves (296.7 \pm 73.8 versus $405.1 \pm 76.6 \mathrm{mmol} \mathrm{l}^{-1} 180 \mathrm{~min}^{-1}$, glucose; $5.022 \pm 1.257$ versus $4.251 \pm 794 \mathrm{mU}^{-1}$ $180 \mathrm{~min}^{-1}$, insulin) were significantly different when insulin was given subcutaneously and intraperitoneally, respectively. However, the occurrence of the peak plasma insulin concentrations following subcutaneous insulin administration (129 $\pm 8 \mathrm{~min})$ was significantly later than that following intraperitoneal insulin administration $(87 \pm 12 \mathrm{~min}, p<0.01)$. The amounts of insulin infused (5.3 \pm 1.7 and $5.5 \pm 0.1$ units, subcutaneous and intraperitoneal, respectively) and the patterns of insulin infusion were not different for the two routes.

The patterns of glucose disposal following the meal were virtually the same during subcutaneous and intraperitoneal insulin administration. Maximal rates of 
glucose appearance $(44.4 \pm 4.4$ versus $49.4 \pm$ $\left.3.9 \mu \mathrm{mol} \cdot \mathrm{kg}^{-1} \cdot \mathrm{min}^{-1}\right)$, disappearance $(49.4 \pm 3.3 \mathrm{ver}-$ sus $\left.49.4 \pm 3.3 \mu \mathrm{mol} \cdot \mathrm{kg}^{-1} \cdot \mathrm{min}^{-1}\right)$ and clearance $(7.2 \pm$ 0.7 versus $6.2 \pm 0.5 \mathrm{ml} \cdot \mathrm{kg}^{-1} \cdot \mathrm{min}^{-1}$ ) and total areas under the curves for glucose appearance $(2.7 \pm 0.3$ versus $\left.2.6 \pm 0.4 \mathrm{mmol} \cdot \mathrm{kg}^{-1} \cdot 180 \mathrm{~min}^{-1}\right)$, disappearance $(2.6$ \pm 0.3 versus $2.7 \pm 0.4 \mathrm{mmol} \cdot \mathrm{kg}^{-1} \cdot 180 \mathrm{~min}^{-1}$ ) and clearance $\left(406 \pm 67\right.$ versus $245 \pm 50 \mathrm{ml} \cdot \mathrm{kg}^{-1} \cdot 180$ $\min ^{-1}$ ) during subcutaneous and intraperitoneal insulin administration were not significantly different.

\section{Discussion}

The current study compared the effects of intraperitoneal and subcutaneous insulin administration on the disposal of glucose in a mixed meal in alloxan diabetic dogs. Our results demonstrate that virtually identical amounts of glucose entered the peripheral circulation following ingestion of the mixed meal with intraperitoneal and subcutaneous insulin administration by a closed-loop device. Since the amounts of glucose utilized were also the same with both routes of insulin administration, plasma glucose concentrations were also equivalent. These observations thus suggest that the hepatic and extrahepatic handling of glucose in the mixed meal did not differ in the dogs when insulin was given by these two routes.

It is well established that the liver is sensitive to small changes in both insulin and glucose concentrations $[27,28]$. It is theoretically possible that, if insulin injected into the peritoneal space were selectively absorbed into the portal venous system, it might act primarily by altering hepatic clearance of glucose. In contrast, since insulin administered via the subcutaneous route is absorbed directly into the peripheral circulation, it may act primarily by accelerating glucose utilization. In addition, since the liver clears approximately $30-50 \%$ of an injected dose of insulin, infusion of insulin into the intraperitoneal space would be expected to result in lower peripheral insulin concentrations than an equivalent amount of insulin injected subcutaneously if there were significant absorption of the intraperitoneal insulin via the portal venous system [29]. Neither of those predictions occurred: rates of glucose appearance and disappearance and circulating plasma insulin concentration were equivalent with both routes.

Shade et al have reported that, following injection of $I^{125}$ insulin into the peritoneal space of anaesthetized recumbent dogs, a greater number of counts per milliliter were observed in the portal vein than in the aorta [30]. These results, although suggesting preferential absorption through the portal vein, are difficult to interpret since portal blood flow is only one-fifth of systemic blood flow. Although the current studies cannot exclude preferential absorption of intraperitoneally injected insulin into the portal venous system, they do indi- cate that if such absorption does occur, its effect on clearance of a mixed meal does not differ from that observed after subcutaneous injection of an equivalent amount of insulin. It should be noted, however, that the current and other studies [16, 18] have demonstrated differences in the kinetics of insulin absorption between these two routes with more rapid absorption occurring from the intraperitoneal route.

The peak post-prandial plasma glucose concentrations in the current study following intraperitoneal or subcutaneous insulin administration $(9-10 \mathrm{mmol} / \mathrm{l}) \mathrm{ex}-$ ceeded those observed in dogs studied under the same conditions when insulin was given by either the portal vein $(7.6 \pm 0.4 \mathrm{mmol} / \mathrm{l})$ or a peripheral vein $(7.2 \pm$ $0.8 \mathrm{mmol} / \mathrm{l}$ ) [31] but were markedly better than those observed in untreated alloxan diabetic dogs given the same meal $(16.1 \pm 2.3 \mathrm{mmol} / \mathrm{l})$ (unpublished observations). It was not the purpose of this study to optimize insulin delivery profiles for the subcutaneous and intraperitoneal routes but rather to examine the patterns of glucose disposal when equivalent insulin infusion rates were given. We cannot exclude the possibility that subtle differences in hepatic and extrahepatic clearance of glucose may have been demonstrable between these two routes of insulin administration if perfect postprandial glycaemic control had been achieved. Furthermore, since the methodology used in the present investigations cannot distinguish between the glucose in the mixed meal and endogenous glucose production, it is possible that differential effects on hepatic glucose uptake versus suppression of endogenous production occurred with the two routes. However, this seems unlikely, since increases in peripheral plasma insulin concentrations equivalent to those observed with both routes of insulin administration in the present study under euglycaemic conditions suppresses hepatic glucose production near totally [27]. The degree of suppression of hepatic glucose production would be expected to at least as great in the present study, since the post-prandial increase in plasma glucose concentration would be expected to enhance the suppressive effect of insulin on glucose production [28]. Nevertheless, even if differences in hepatic uptake of glucose and suppression of hepatic glucose production did occur, the net amount of glucose reaching the peripheral circulation following a mixed meal did not differ when insulin was administered subcutaneously and intraperitoneally.

Thus, although the kinetics of insulin absorption using the intraperitoneal route may differ from those observed using the subcutaneous route, the current studies demonstrate that in the alloxan diabetic dog there appears to be no difference in the pattern of disposal of glucose from a mixed meal when insulin is administered intraperitoneally or subcutaneously with the same algorithm by a closed-loop insulin infusion device. Whether this would also occur following intraperitoneal and subcutaneous insulin delivery in diabetic man remains to be determined. 
Acknowledgements. The excellent technical assistance of K.Greene, B. Brock, J. King, D.Stenner and W. Blanchard is gratefully acknowledged. In addition, we are indebted to Sue Sturgis for her skillfull editorial assistance in the preparation of this manuscript. This project was funded in part by USPHS grants AM 82218, AM 00648, AM 20411, AM 20837 and AM 5827.

\section{References}

1. Slama G, Hautecouverture M, Assan R, Tchobroutsky G (1974) One to five days of continuous intravenous insulin infusion on seven diabetic patients. Diabetes 23: 732-738

2. Hepp KD, Renner R, Funcke HG, Mehnert H, Hoerten R, Kreese H (1977) Glucose homeostasis under continuous intravenous insulin therapy in diabetes. Horm Metab Res (Suppl 7): $72-76$

3. Service FJ (1978) Normalization of plasma glucose of unstable diabetes: Studies under ambulatory fed conditions with pumped intravenous insulin. J Lab Clin Med 91: 480-489

4. Bojsen J, Deckert T, Kolendorg K, Lorup B (1979) Patient-controlled portable insulin infusion pump in diabetics. Diabetes 28 : 974-979

5. Hanna AK, Minuk HL, Albisser AM, Marliss EB, Leibel B, Zinman B (1980) A portable system for continuous intravenous insulin delivery: Characteristics and results in human diabetics. Diabetes Care 3: $1-8$

6. Pickup JC, Keen H, Parsons JA, Alberti KGMM (1978) Continuous subcutaneous insulin infusion: An approach to achieving normoglycaemia. Br Med J 1: 204-207

7. Tamborlane WV, Sherwin RS, Genel M, Felig P (1979) Reduction to normal of plasma glucose in juvenile diabetes by subcutaneous administration of insulin with a portable infusion pump. $\mathrm{N}$ Engl $\mathbf{J}$ Med 300: 573-578

8. Rizza RA, Gerich JE, Haymond MW, Westland RE, Hall LD, Clemens AH, Service FJ (1980) Control of blood sugar in insulindependent diabetes: Comparison of an artificial endocrine pancreas, continuous subcutaneous insulin infusion and intensified conventional insulin therapy. N Engl J Med 303: 1313-1318

9. Pickup JC, White MC, Keen H, Parsons JA, Alberti KGMM (1979) Long-term continuous subcutaneous insulin infusion in diabetics at home. Lancet 2: 870-873

10. Champion MC, Shephert GAA, Rodger NW, Dupre J (1980) Continuous subcutaneous infusion of insulin in the management of diabetes mellitus. Diabetes 29: 206-212

11. Tamborlane WV, Sherwin RS, Genel M, Felig P (1980) Outpatient treatment of juvenile-onset diabetes with a preprogrammed portable subcutaneous insulin infusion system. Am J Med 68: 190-196

12. Raskin P, Pietri A, Unger R (1979) Changes in glucagon levels after four to five weeks of glucoregulation by portable insulin infusion pumps. Diabetes 28: 1033-1035

13. Botz C, Leibel B, Zing W, Gander R, Albisser A (1976) Comparison of peripheral and portal routes of insulin infusion by a computer-controlled insulin infusion system (artificial endocrine pancreas). Diabetes 25: 691-700

14. Schade DS, Eaton RP, Spencer W, Goldman R, Corbett WT (1979) The peritoneal absorption of insulin in diabetic man: A potential site for a mechanical insulin delivery system. Metabolism 28: 195-197

15. Schade DS, Eaton RP, Friedman NW, Spencer W (1980) Pro- longed peritoneal insulin infusion in a diabetic man. Diabetes Care 3: 314-317

16. Schade DS, Eaton RP, Friedman NW, Spencer W (1979) The intravenous, intraperitoneal and subcutaneous routes of insulin delivery in diabetic man. Diabetes 28: 1069-1072

17. Irsigler K, Kritz H (1980) Alternate routes of insulin delivery. Diabetes Care 3: 219-228

18. Schade DS, Eaton RP, Friedman NW, Spencer W (1980) Normalization of plasma insulin profiles with intraperitoneal insulin infusion in diabetic man. Diabetologia 19: 35-39

19. Herbert V, Lau K, Gottleib C, Bleicher S (1965) Coated charcoal immunoassay of insulin. J Clin Endocr Metab 25: 1375-1384

20. Rizza RA, Cryer P, Gerich JE (1979) Role of glucagon, catecholamine and growth hormone in human glucose counterregulation. Effect of somatostatin and combined alpha and beta adrenergic blockade on plasma glucose recovery and glucose flux rates after insulin-induced hypoglycemia. J Clin Invest 64: 62-71

21. Steele R, Wall J, De Bodo R, Altszuler N (1956) Measurement of size and turnover rate of body glucose pool by the isotope dilution method. Am J Physiol 187: 15-24

22. De Bodo R, Steele R, Altszuler N, Dunn A, Bishop J (1963) On the hormonal regulation of carbohydrate metabolism: Studies with $\mathrm{C}^{14}$ glucose. Rec Prog Horm Res 19: 445-488

23. Radziuk J, Norwich KH, Vranic M (1978) Experimental validation of measurements of glucose turnover in nonsteady state. Am J Physiol 234 (1): E 84-E93

24. Riggs L (1963) The mathematical approach to physiologic problems. Williams and Wilkins, Baltimore

25. Altszuler W, Barkai A, Bjerkness C, Gottleib B, Steele R (1975) Glucose turnover values in dogs obtained with various species of labeled glucose. Am J Physiol 229: 1662-1667

26. Zar J (1974) Biostatistical analysis. Prentice Hall, Englewood Cliffs, New Jersey

27. Rizza RA, Mandarino LJ, Gerich JE (1981) Dose response characteristics for the effects of insulin on glucose production, glucose utilization and overall glucose metabolism in man: Determination using sequential infusions of insulin in conjunction with the glucose clamp technique. Am J Physiol 3: E630-639

28. Bergman RN, Buedo RJ (1974) Interaction of insulin and glucose in the control of hepatic glucose balance. Am J Physiol 227: $1314-1322$

29. Field JB (1972) Insulin extraction by the liver. In: Steiner DF, Freinkel N (eds) Handbook of physiology: A critical comprehensive presentation of physiological knowledge and concepts, vol 1, section 7. American Physiological Society, Washington, D.C., pp 505-513

30. Schade DS, Eaton RP, Davis T, Akiya F, Phinney E, Kubica R, Vaugh EA, Day PW (1981) The kinetics of peritoneal insulin absorption. Metabolism 30: 149-155

31. Rizza RA, Westland RE, Hall LD, Patton GS, Haymond MW, Clemens AH, Gerich JE, Service FJ (1981) Effect of peripheral versus portal venous administration of insulin on post-prandial hyperglycemia and glucose turnover in alloxan diabetic dogs. Mayo Clin Proc 56: 434-438

Received: 21 August 1981

and in revised form: 26 January 1982

Dr. R.A. Rizza

Mayo Clinic

Rochester, Minnesota 55901, USA 